\title{
Empirical Scaling Relations
}

\author{
Tommaso Treu \\ Department of Physics, University of California, Santa Barbara, CA 93106-9530, USA \\ email: tt@physics.ucsb.edu
}

\begin{abstract}
Early-type galaxies do not come in any shape, form, and color. Many of their observable properties obey tight correlations, also known as empirical scaling relations. The correlations are non-trivial, in the sense that they cannot be explained by simple physical or dimensional arguments. A subset of the empirical scaling relations connects baryonic observables with quantities that depend on the total gravitational potential of the galaxies, and thus on their dark matter content. These correlations are a fundamental testbed for our understanding of the formation and evolution of early-type galaxies, and, more in general, of the physical processes that determine the interplay between baryons and dark matter at galactic scales.
\end{abstract}

Keywords. Galaxies: elliptical and lenticular, cD - Galaxies: formation - Galaxies: evolution

The fundamental plane Djorgovski \& Davis (1987), Dressler et al. (1987), the more fundamental plane Bolton et al. (2007) - together with their projections and their extension including stellar mass estimators - connect their baryonic content to their dark matter content. One key feature of these scaling relations is the so-called "tilt" of the fundamental plane (e.g. Ciotti et al. (1996)), i.e. the fact that M/L increases with galaxy stellar mass. The "tilt" could be the result of several competing effects, the main suspects being systematic changes in internal dynamical structure with galaxy size and stellar population changes. Recent studies (see also Cappellari, this meeting), indicate that the trends are due in some part to an increase in stellar mass to light ratio with stellar mass, resulting from the now well-established mass metallicity and mass age correlations (also known as downsizing). However, these are not sufficient, and a systematic increase in dark matter content (or stellar IMF) with stellar mass appears necessary to full reproduce the "tilt" Bolton et al. (2008), Treu et al. (2009).

These trends are particularly remarkable if one considers the tight scatter of the scaling relations. In fact, their tightness is an extremely powerful constraint on galaxy evolution models. Nipoti et al. (2009) showed that although dry mergers could be invoked to explain the apparent growth in size of early-type galaxies since $z \sim 2$, they tend to destroy the observed correlations. Therefore, the progenitors of present day early-type galaxies cannot have formed from galaxies obeying the same scaling relations. Likewise, early-type galaxies are unlikely to have accreted more than half of their current mass by dry mergers.

\section{References}

Bolton, A. S. et al. 2008, ApJ, 684, 248

Bolton, A. S. et al. 2007 ApJL, 665, L105

Djorgovski, S. \& Davis, M. 1987, ApJ, 313, 59

Ciotti, L., Lanzoni, B., \& Renzini, A. 1996, MNRAS, 282, 1

Dressler, A., et al. 1987, ApJ, 313, 42

Nipoti, C., Treu, T., Auger, M. W., \& Bolton, A. S. 2009, ApJL, 706, L86

Treu, T. et al. 2009, ApJ, submitted, arXiv:0911.3392 